\title{
Rare cause of gastric outlet obstruction
}

\author{
Sonal Gandhi ${ }^{\mathrm{a}}$ and Niraj Jani ${ }^{\mathrm{b}}$
}

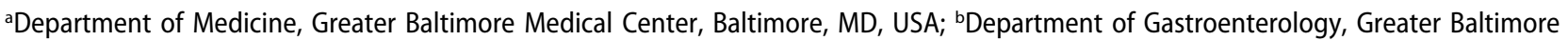
Medical Center, Baltimore, MD, USA

\section{ABSTRACT}

Bouveret's syndrome is a rare cause of gastric outlet obstruction. The stones enter the small bowel via cholecysto-enteric fistula. The most common presenting symptoms are abdominal pain, nausea and vomiting. The gold standard diagnostic test is esophagogastroduodenoscopy (EGD). Rigler's triad on abdominal $x$-ray is classic. CT scan findings are pneumobilia, cholecystoduodenal fistula and a gallstone in the duodenum. We present a case of a 75-yearold female who presents with 3 week history of nausea, vomiting, and diffuse abdominal pain. Initial presentation, imaging and EGD was concerning for malignancy. She was later diagnosed to have Bouveret's syndrome and underwent laparoscopic small bowel enterotomy with removal of gallstones
ARTICLE HISTORY

Received 24 October 2017 Accepted 8 March 2018

\section{KEYWORDS}

Abdominal pain; cholecystoenteric fistula; Rigler's triad; pneumobilia; Bouveret's syndrome
Patient is a 75-year-old female who presented with a 3week history of nausea, vomiting and diffuse abdominal pain and distension. She reported her pain to be intermittent, sharp, 3/10 in severity and located in the epigastric area. She was noted to be in Atrial Fibrillation on presentation but other vital signs were normal. Patient's past medical history was significant for Diabetes Mellitus, she was not on any medication and has not seen a physician in many years. Her only past surgical history included repair of an atrial septal defect at the age of 18 .

Laboratory data on admission revealed WBC of $11 \mathrm{k}$, sodium 146, anion Gap 22, Glglucose 334 and HbA1c of 9.2. liver function tests, amylase, lipase and lactic acid were noted to be normal.

On physical exam, her abdomen was distended and tender in the RUQ. A CT scan of the abdomen and pelvis revealed marked dilation of the stomach with fluid, pneumobilia, and poor gallbladder visualization. (Figure 1) The patient did not have any history of an abdominal surgery or Endoscopic Retrograde Cholangiopancreatography (ERCP). The patient was started on IV Fluids, IV PPI and was made NPO. The NG tube was also inserted and was put to low intermittent suction.

Esophagogastroduodenoscopy (EGD) showed Grade $\mathrm{D}$ esophagitis, excessive fluid in stomach and a large ulcerated area in second part of duodenum. (Figure 2) There was a large brown hard object embedded within the ulcer which could be not be removed. Biopsies were negative for malignancy, it revealed ulcerated duodenal mucosa with acute and chronic inflammatory changes and scarring. A PET scan was suggestive of acute cholecystitis versus a cholecystoenteric fistula or malignancy.

Repeat EGD a week later showed total resolution of the ulcerated area in the duodenum and a significant improvement in esophagitis to LA Grade A. (Figure 3) EUS revealed peri-pancreatic inflammation and poor visualization of the gallbladder. Repeat CT scan revealed dilated proximal jejunal small bowel loops with transition zone and a $1.5 \mathrm{~cm}$ rounded structure in the jejunum.

The patient was thought to have chronic cholecystitis likely from sub-acute to chronic inflammation of the gall bladder from the underlying gallstone disease causing perforation of the gallbladder with formation of a cholecystoenteric fistula resulting in gallstone impaction and small bowel obstruction. She underwent laparoscopic small bowel enterotomy with removal of gallstones from distal jejunum and proximal ileum. (Figure 4) The patient slowly began eating and was discharged with uneventful post-operative course.

\section{Discussion}

Bouveret's syndrome is a rare cause of gastric outlet obstruction. Gallstone ileus is seen in $1 \%-4 \%$ of small bowel obtruction, and involves duodenum in only $1 \%-3 \%$ of cases [1]. It is a variant of gallstone ileus resulting in gastroduodenal obstruction secondary to gallstone impaction in the duodenum. The stones enter the small bowel via cholecysto-enteric fistula which is created by chronic inflammation of the adherent biliary system and bowel walls causing necrosis [2]. Bouveret's syndrome is usually caused

CONTACT Sonal Gandhi sgandhi@gbmc.org Department of Medicine, Greater Baltimore Medical Center, Baltimore, MD, USA

Bouveret's syndrome was first described in 1841 by Bonnet. The first published report of Bouveret's syndrome (1896) is attributed to Leon Bouveret- a French internist, who reported on two patients with this disease 


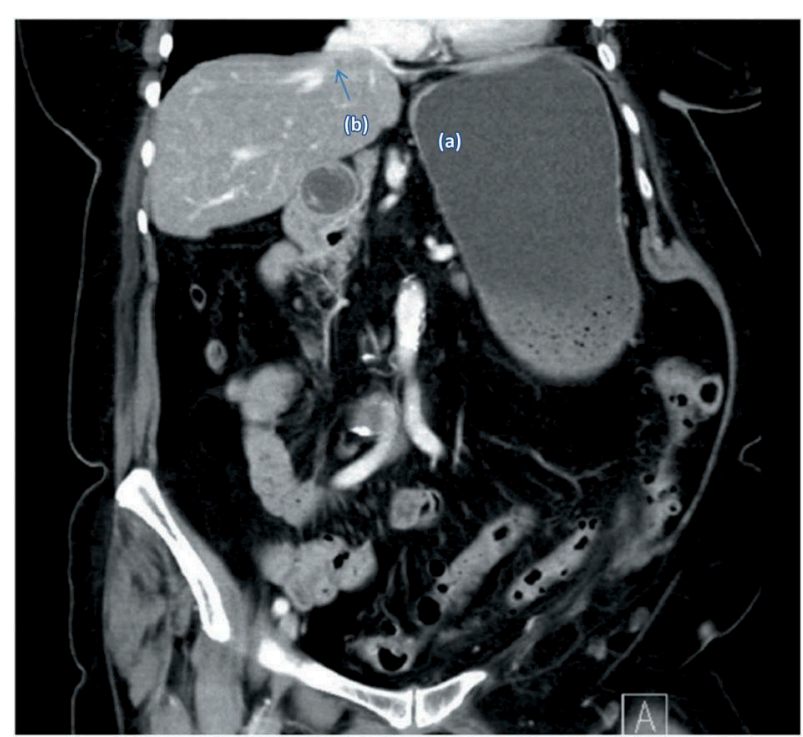

Figure 1. CT Abdomen Pelvis upon presentation. (a) Marked distention of the stomach with fluid. (b) Pneumobilia involving left lobe of liver. (Arrow pointing). by stones that are larger than stones that cause gallstone ileus. The average size is $4.6 \mathrm{~cm}$ compared to $2.5 \mathrm{~cm}$ [2]. It complicates $0.3 \%-5 \%$ of cholelithiasis $[2,3]$.

The most common presenting symptoms are abdominal pain, nausea and vomiting. Other non-specific symptoms include hematemesis, weight loss, anorexia and duodenal perforation [1-14]. Differential Diagnosis include pancreatitis, perforated peptic ulcer, malignant fistula, gastric volvulus [4,5,12-14].

Diagnosis depends on high index of clinical suspicion along with imaging studies [15]. The gold standard diagnostic test is EGD although CT scan, abdominal ultrasound and plain $\mathrm{x}$-ray can be very helpful. Rigler's triad of pneumobilia, an ectopic gallstone, and dilated small bowel on abdominal $\mathrm{x}$-ray is classic but is only seen in $30 \%-35 \%$ of cases [6]. Classic CT scan findings are pneumobilia, cholecystoduodenal fistula and a gallstone in the duodenum $[2,3,15]$. Although, $15 \%-25 \%$ of gallstones
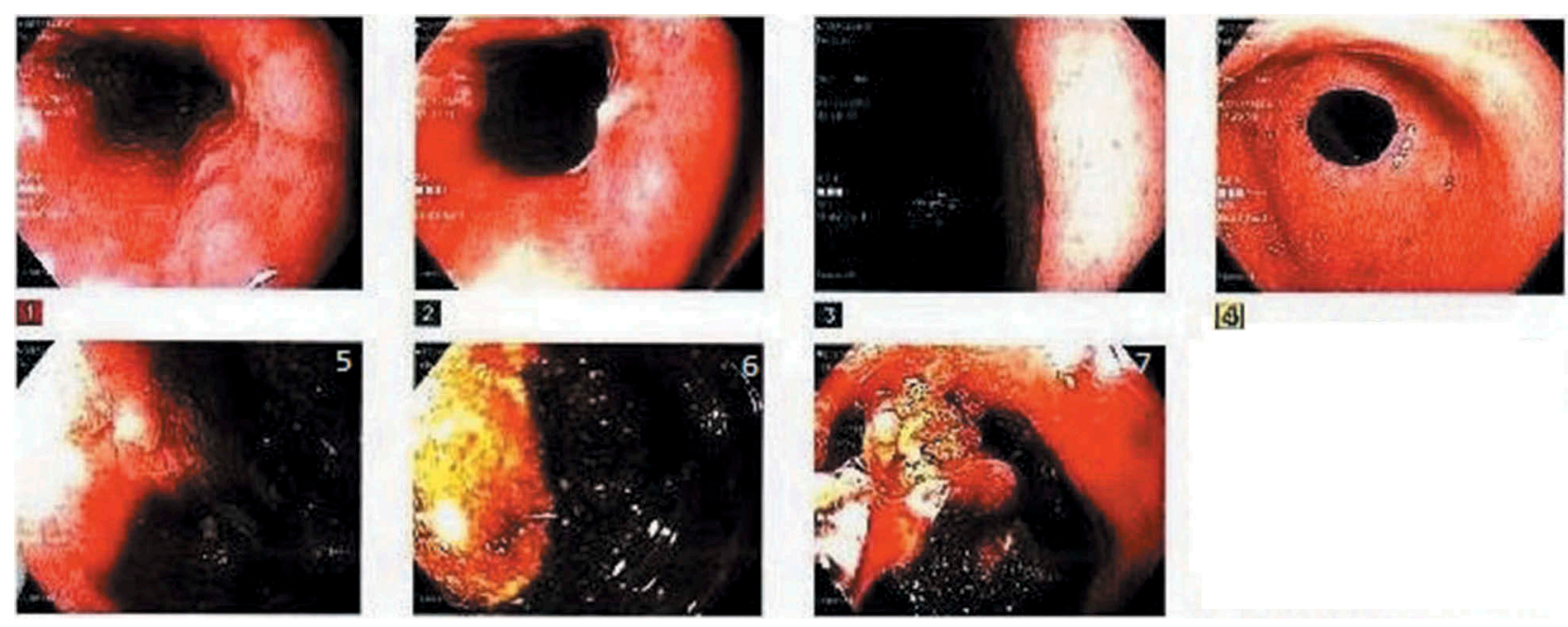

[ब]

Figure 2. Initial EGD 1, 2-Reveal LA grade D esophagitis 6, 7-Reveal large malignant ulcerated mass with stigmata of bleeding in second part of duodenum.
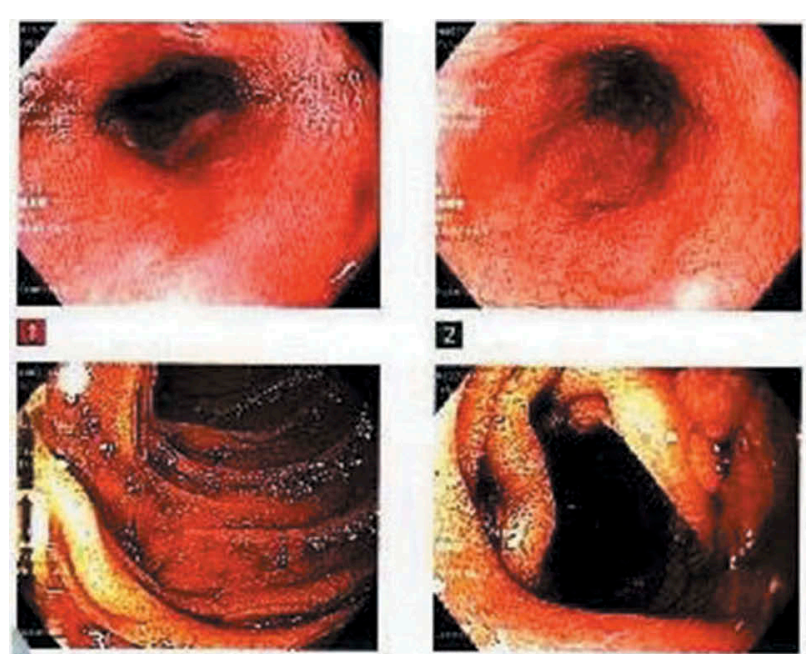

田

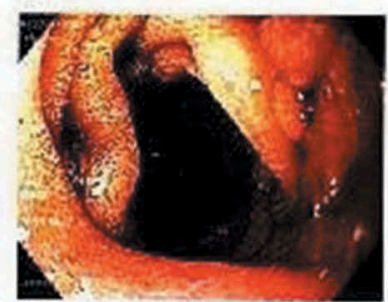

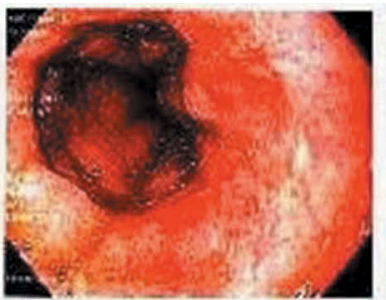

3.

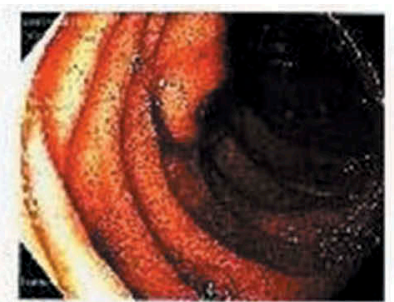

[द]

Figure 3. Repeat EGD 1 week later. 3-Reveal LA grade A erosive gastritis 5, 6-Reveal complete resolution of prior duodenal ulcer. 


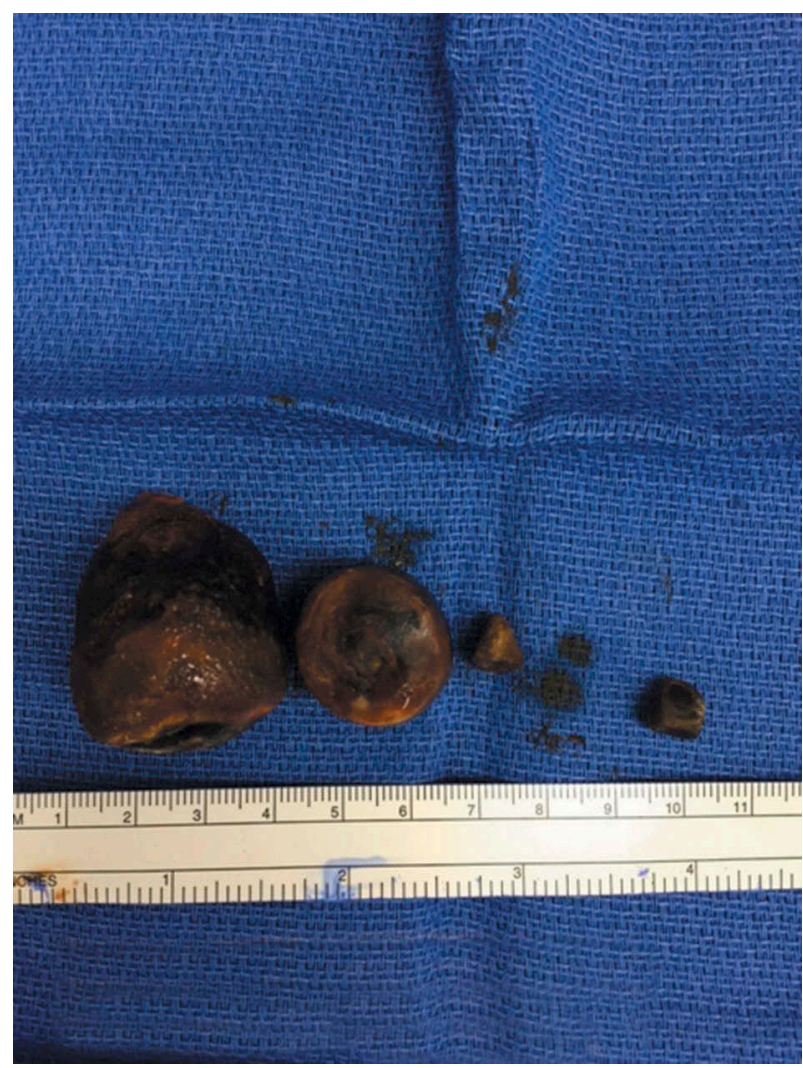

Figure 4. Multiple gallstones removed from distal jejunum and proximal ileum through laparoscopic small bowel enterotomy.

cannot be visualized on CT scan as they can be isoattenuating. In those cases MRCP can help with diagnosis. EGD can be both diagnostic and therapeutic but success rate for stone removal is low.

Although the gastric outlet obstruction is almost always seen on EGD, the obstructing gallstone is seen only in $69 \%$ of cases [2].

Treatment options include endoscopic removal, open surgery or laparoscopic removal of the stones. Endoscopic procedures are always attempted first with a success rate of less than $10 \%$ [5]. It can be accompanied by lithotripsy but it can cause the stone fragments to migrate distally causing gallstone ileus. Endoscopic options are tried first as majority of patients are elderly and poor surgical candidates. Gallstones that are larger than $2.5 \mathrm{~cm}$ are difficult to remove endoscopically, can result in esophagus impaction or distal impaction if they are broken into fragments [5]. Laparoscopic and open surgical options are then explored. Surgery is the mainstay for treatment consisting of enterolithotomy and gastrotomy [16]. Cholecystectomy is recommended to prevent disease recurrence [7].

Our patient initially presented with Bouveret's syndrome which was then complicated by gallstone ileus. Her risk for gallstone was likely from her gender and long standing untreated diabetes. She was found to have several large stones causing small bowel obstruction. Our initial suspicion was more for malignancy prompting the PET CT scan that was suggestive of cholecystoenteric fistula.

\section{Disclosure statement}

No potential conflict of interest was reported by the authors.

\section{References}

[1] Gallego Otaegui L, Sainz Lete A, Gutiérrez Ríos RD, et al. A rare presentation of gallstones: Bouveret's syndrome, a case report. Rev Esp Enferm Dig. 2016 Jul;108(7):434-436.

[2] Nickel F, Muller-Eschner MM, Chu J, et al. Bouveret's syndrome: presentation of two cases with review of the literature and development of a surgical treatment strategy. BMC Surg. 2013 Sep 4;13:33-2482-13-33.

[3] Ayantunde AA, Agrawal A. Gallstone Ileus: diagnosis and management. World J Surg. 2007 Jun;31(6):1292-1297.

[4] Bonam R, Vahora Z, Harvin G, et al. Bouveret's syndrome with severe esophagitis and a purulent fistula. ACG Case Rep J. 2014 Apr 4;1(3):158-160.

[5] Doycheva I, Limaye A, Suman A, et al. Bouveret's syndrome: case report and review of the literature. Gastroenterol Res Pract. 2009;2009:914951.

[6] Gaduputi V, Tariq H, Rahnemai-Azar AA, et al. Gallstone ileus with multiple stones: where Rigler triad meets Bouveret's syndrome. World J Gastrointest Surg. 2015 Dec 27;7(12):394-397.

[7] Jafferbhoy S, Rustum Q, Shiwani M. Bouveret's syndrome: should we remove the gall bladder? BMJ Case Rep. 2011 Apr 1;2011. DOI:10.1136/bcr.02.2011.3891

[8] Kalwaniya DS, Arya SV, Guha S, et al. A rare presentation of gastric outlet obstruction (GOO) - The Bouveret's syndrome. Ann Med Surg (Lond). 2015 Feb 16;4(1):67-71.

[9] Mavroeidis VK, Matthioudakis DI, Economou NK, et al. Bouveret syndrome-the rarest variant of gallstone ileus: a case report and literature review. Case Rep Surg. 2013;2013:839370.

[10] Qasaimeh GR, Bakkar S, Jadallah K. Bouveret's syndrome: an overlooked diagnosis. A case report and review of literature. Int Surg. 2014 Nov-Dec;99(6):819-823.

[11] Reinhardt SW, Jin LX, Pitt SC, et al. Bouveret's syndrome complicated by classic gallstone ileus: progression of disease or iatrogenic? J Gastrointest Surg. 2013 Nov;17(11):2020-2024.

[12] Zafar A, Ingham G, Jameel JK. "Bouveret's syndrome” presenting with acute pancreatitis a very rare and challenging variant of gallstone ileus. Int J Surg Case Rep. 2013;4 (5):528-530.

[13] Gencosmanoglu R, Inceoglu R, Baysal C, et al. Bouveret's syndrome complicated by a distal gallstone ileus. World J Gastroenterol. 2003 Dec;9(12):2873-2875.

[14] Cappell MS, Davis M. Characterization of Bouveret's syndrome: a comprehensive review of 128 cases. Am J Gastroenterol. 2006 Sep;101(9):2139-2146.

[15] Toth E, Zawadski A, Thorlacius H. Education and imaging: gastrointestinal: cholecystoduodenal fistula with gallstone-induced intestinal obstruction. J Gastroenterol Hepatol. 2013 Feb;28(2):377.

[16] Hussain A, Obaid S, El-Hasani S. Bouveret's syndrome: endoscopic or surgical treatment. Updates Surg. 2013 Mar;65(1):63-65. 